\title{
On the torsional loading of elastoplastic spheres in contact
}

\author{
Sadegh Nadimi $^{1, *}$, and Joana Fonseca ${ }^{1}$ \\ ${ }^{1}$ City, University of London, London, UK
}

\begin{abstract}
The mechanical interaction between two bodies involves normal loading in combination with tangential, torsional and rotational loading. This paper focuses on the torsional loading of two spherical bodies which leads to twisting moment. The theoretical approach for calculating twisting moment between two spherical bodies has been proposed by Lubkin [1]. Due to the complexity of the solution, this has been simplified by Deresiewicz for discrete element modelling [2]. Here, the application of a simplified model for elastoplastic spheres is verified using computational modelling. The single grain interaction is simulated in a combined finite discrete element domain. In this domain a grain can deform using a finite element formulation and can interact with other objects based on discrete element principles. For an elastoplastic model, the contact area is larger in comparison with the elastic model, under a given normal force. Therefore, the plastic twisting moment is stiffer. The results presented here are important for describing any granular system involving torsional loading of elastoplastic grains. In particular, recent research on the behaviour of soil has clearly shown the importance of plasticity on grain interaction and rearrangement.
\end{abstract}

\section{Background}

Mathematical models have been incorporated into discrete modelling of granular system describing the force-displacement relationship between two contacting rigid bodies. This has proven the reliability in simulating granular materials such as soil $[3,4]$. The interaction includes normal contact with relative contact area motions such as sliding, rolling or spinning. The spinning around the axis of the contact normal creates a twisting moment $\left(M_{T}\right)$. When $M_{T}$ in combination with normal loading is applied to two grains in contact, the contact area will undergo some angular displacement $(\beta)$. The shear forces at the contact will provide some resistance to sliding. Depending on the distribution of normal forces, the region that meets the Coulomb friction condition will experience sliding and the rest of the contact area will undergo sticking $[5,6]$. The schematic of this mixed boundary problem for two identical spheres in contact where stick and slip regions coexist is shown in Fig. 1.

The relationship between the applied moment and the radius of stick region can be written as follows:

$M_{T}=2 \pi \int_{0}^{a} q(r) r^{2} d r$

where $q(r)$ is torsional shear traction that is a function of $r$, the radial position from centre of the contact area. If $c$ $\leq r \leq a$, the traction is limited to $q(r)=\mu F_{N}(r)$.

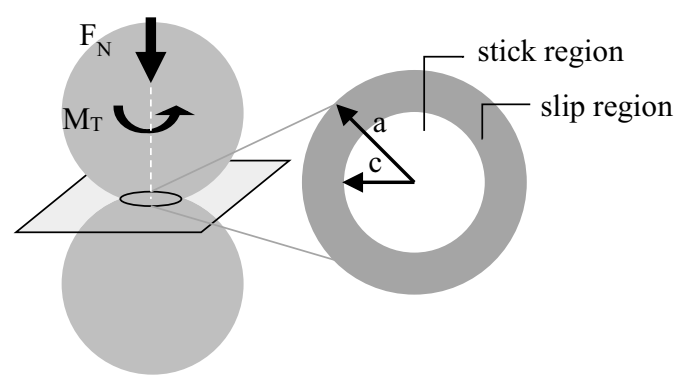

Fig. 1. Contact area including stick and slip regions for two identical spheres subjected to torsional moment and normal loading.

Lubkin (1951) delivers the solution to this problem by proposing an equation to define the shear stress at the contact surface within the stick region [1]. By combining Lubkin's solution with normal force distribution, the twisting moment can be obtained from the following expression $[1,5]$ :

$$
\begin{aligned}
& M_{T}=\frac{\mu F_{N} a}{4 \pi} \times \\
& \left\{\begin{array}{l}
\frac{3 \pi^{2}}{4}+k^{\prime} k^{2}\left[6 K(k)+\left(4 k^{\prime 2}-3\right) D\right]-3 k K(k) \sin ^{-1} k^{\prime}-3 k^{2} \\
{\left[K(k) \int_{0}^{\pi / 2} \frac{\sin ^{-1}\left(k^{\prime} \sin \alpha\right) d \alpha}{\left(1-k^{\prime 2} \sin ^{2} \alpha\right)^{3 / 2}}-D(k) \int_{0}^{\pi / 2} \frac{\sin ^{-1}\left(k^{\prime} \sin \alpha\right) d \alpha}{\left(1-k^{\prime 2} \sin ^{2} \alpha\right)^{1 / 2}}\right]}
\end{array}\right]
\end{aligned}
$$

\footnotetext{
* Corresponding author: sadegh.nadimi-shahraki@,city.ac.uk
} 
where $k=\sqrt{1-(c / a)^{2}}, \quad k^{\prime}=\frac{c}{a}$ and $\sin \alpha=r / c$. $D(k)$ is the complete elliptical integral with modulus $K$, given by $D(k)=(K-E) / k 2$ with $K$ and $E$ being the complete elliptical integrals of the first and second type, respectively.

Given the complexity of Eq. 1, a simplified solution was proposed by Deresiewicz (1954) between $a, M_{T}$ and $\beta$ based on an explicit approximation for numerical modelling which is defined as follows:

$$
\frac{G a^{2} \beta}{\mu F_{N}}=\frac{1}{8}\left[1-\sqrt{1-\frac{3}{2} \frac{M_{T}}{\mu F_{N} a}}\right] \times\left[3-\sqrt{1-\frac{3}{2} \frac{M_{T}}{\mu F_{N} a}}\right]
$$

where $G$ is the shear modulus. Therefore, the torsional stiffness can be specified as:

$$
k_{t}=\frac{d M_{T}}{d \beta}=\frac{16 G a^{3}}{3}\left[2\left(1-\frac{3}{2} \frac{M_{T}}{\mu F_{N} a}\right)^{-1 / 2}-1\right]^{-1}
$$

It is important to note that the Eqs. 3 and 4 are only applicable for small values of twisting moment where $M_{T} / \mu F_{N} a<<1$ [5]. Fig. 2 shows a non-dimensional moment-twist profile derived from Eq. 3.

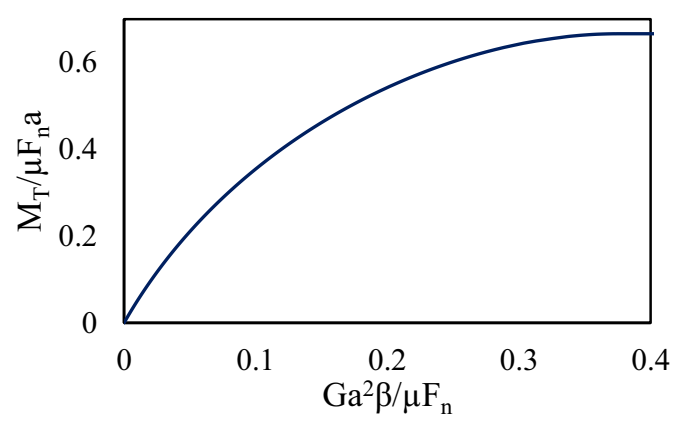

Fig. 2. Non-dimensional relationship between torque and twisting angle for spherical grains.

Despite the development of this theoretical approach for the elastic interaction of spheres in the fifties, the elastoplastic interaction under torsion has not been verified yet, as discussed in Thornton [4].

In this paper, the problem is replicated in the framework of a finite-discrete element method [7]. In this framework, a continuum body can deform using finite element formulation and can interact with other objects (rigid and deformable) based on the law of motion, i.e. Newton's second law, used in discrete element modelling. Firstly, the elastic behaviour of a sphere in contact is verified against theoretical equations. Then, the elastoplastic behaviour is presented. The aim of this study is to investigate the applicability of Deresiewicz's solution for elastoplastic grains in contact under twisting moment. The results have implications for describing a granular system with elastoplastic grains.

\section{Elastic interaction}

The interaction of two identical spheres in contact is simplified, due to symmetry, by the interaction of a sphere in contact with a rigid plate. In order to apply pure torsion to a deformable sphere, a rigid core was generated inside the sphere and was tied to the sphere (Fig. 3). Fig. 4 shows the numerical mesh of the problem in Abaqus software package [8]. The sphere has a diameter of $2.2 \mathrm{~mm}$ and is represented by a mesh formed by 60,743 elements and 18,112 nodes. The mesh at the contact area was refined for a more accurate presentation of the problem. The material parameters used in the simulation are listed in Table 1. Explicit time discretization was employed to allow for future work on a large number of grains, since the implicit time discretization is computationally very expensive. The property of hard contact was defined between the sphere and the plate. Using 'hard contact' behaviour means that all the force is transmitted through the contact. Due to body deformability, the relation of normal force versus normal displacement with hard contact assumption follows exactly Hertzian theory [6, 9].

The simulation includes two steps: normal loading and torsional loading. In step one, controlled displacement of $10 \mu \mathrm{m}$ was applied to the sphere in the normal direction, which corresponds to $95 \mathrm{~N}$ normal loading for this problem. In the second step, the sphere was purely rotated around the contact normal using controlled angular displacement of $0.04 \mathrm{rad}$.

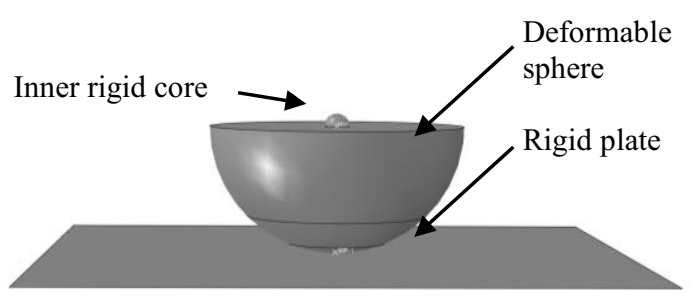

Fig. 3. Schematic showing the inner core and cut section of the deformable sphere.

Table 1. Physical and mechanical parameters of the spheres.

\begin{tabular}{|c|c|c|}
\hline Elastic modulus & E (GPa) & 63 \\
\hline Poisson ratio & $v(-)$ & 0.3 \\
\hline Density & $\rho\left(\mathrm{gr} / \mathrm{mm}^{3}\right)$ & 2.5 \\
\hline Friction coefficient & $\mu(-)$ & 0.22 \\
\hline Diameter & $\mathrm{D}(\mathrm{mm})$ & 2.2 \\
\hline
\end{tabular}




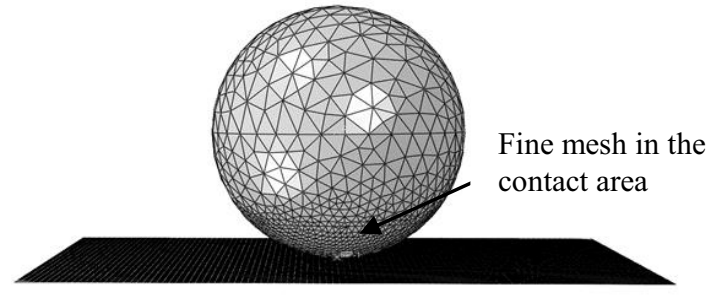

Fig. 4. Meshed sphere in contact with the rigid plate; mesh defined by smaller elements in the contact area.

The comparison between Deresiewicz theory and the numerical simulation is presented in Fig. 5. The plot shows a good agreement between the numerical model and theory. As can be seen in Fig. 5, there is a small discrepancy in the angular displacement $(\beta)$ corresponding to the occurrence of pure slipping.

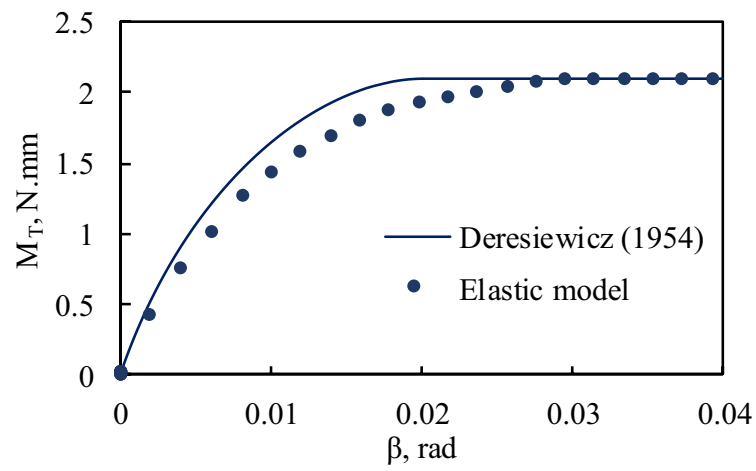

Fig. 5. Comparison between theory and numerical modelling for elastic interaction of two identical elastic spheres.

\section{Elastoplastic interaction}

The torsional interaction becomes plastic when the yield strength is reached in normal loading. This is particularly important for soil grains due to initial contact plasticity [4,9]. The contact area would be larger for elastoplastic interaction in comparison with elastic interaction for a given normal load. Therefore, pure shear force shows higher tangential stiffness [4]. It is also expected to observe higher stiffness $\left(k_{t}\right)$ due to the larger radius of contact area $(a)$ in torsional loading.

To incorporate the plastic behaviour in numerical modelling, isotropic hardening constitutive laws were assigned to the sphere. It is assumed that the material yields at $100 \mathrm{MPa}$ and then hardens with hardening modulus of $20 \mathrm{GPa}$ (Table 2). The stress:strain relation assigned to the material is shown in Fig. 6 .

The simulation steps were exactly the same as for the elastic model. In order to keep the normal load of $95 \mathrm{~N}$, a $47 \mu \mathrm{m}$ controlled displacement was applied in the normal direction which changes the ' $a$ ' value from $149 \mu \mathrm{m}$ (for elastic) to $319 \mu \mathrm{m}$ for plastic interaction. This was obtained by trial and error. In the second step, controlled angular displacement of $0.04 \mathrm{rad}$ was applied to the sphere around the contact normal.

Table 2. Isotropic hardening parameters used.

\begin{tabular}{|c|c|c|}
\hline Yield strength & Y (MPa) & 100 \\
\hline Hardening modulus & Et (GPa) & 20 \\
\hline
\end{tabular}

Fig. 7 shows the comparison of the numerical simulations for elastic and elastoplastic interaction in terms of twisting moment and angular displacement. It can be seen that the value of plastic twisting moment is 2.5 times the elastic twisting moment for a constant normal loading, while the contact area was nearly doubled. The normalised twisting moment, presenting in Fig. 8, shows that the plastic interaction is stiffer than the elastic interaction and pure slipping occurs at smaller values of angular displacement. Finally, the comparison of the theoretical, elastic and plastic models for normalised twisting moment versus normalised angular displacement are presented in Fig. 9. The good agreement observed, suggests the applicability of Deresiewicz solution for elastoplastic interaction.

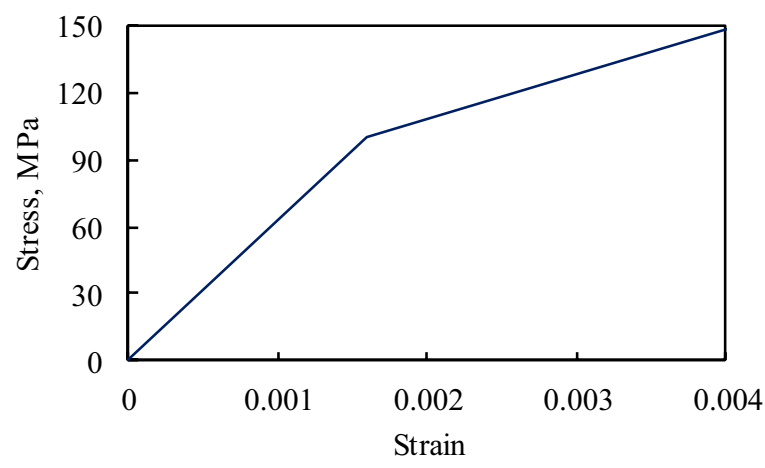

Fig. 6. Stress:strain for isotropic hardening material used in the simulation.

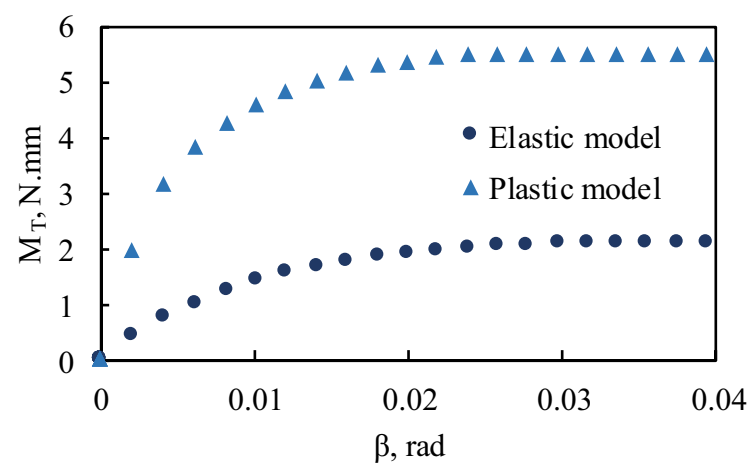

Fig. 7. Comparison between the numerical modelling of elastic and plastic interaction of two identical spheres under torsion and constant normal loading. 


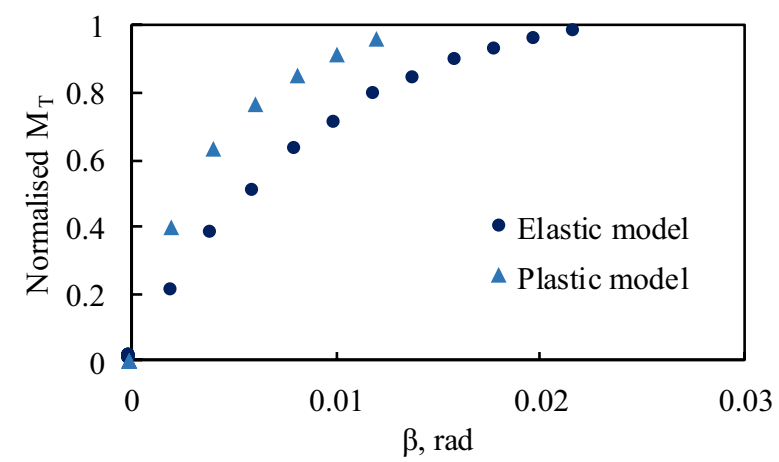

Fig. 8. Comparison between the normalised twisting moments derived from numerical modelling of elastic and plastic interaction of two identical spheres under torsion and constant normal loading.

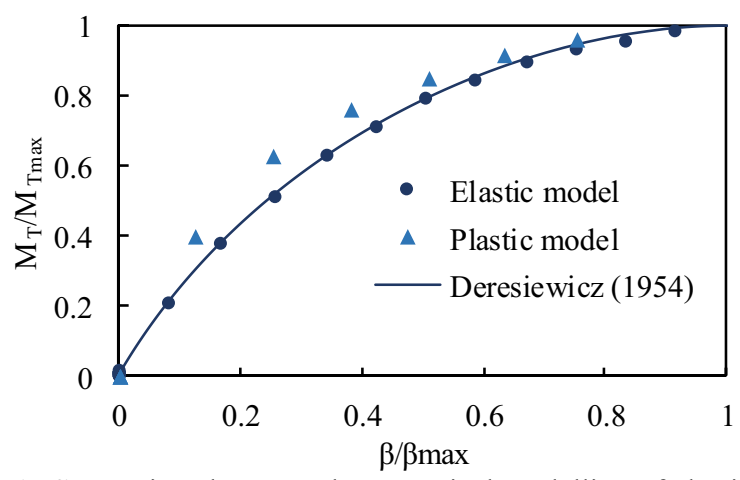

Fig. 9. Comparison between the numerical modelling of elastic and plastic interaction and Deresiewicz's solution of two identical spheres under torsion and constant normal loading.

\section{Closing remarks}

This study makes use of the general contact model for two identical spherical grains twisted around their contact normal as proposed by Lubkin and later simplified by Deresiewicz. The formulations proposed were verified for elastic and more importantly for elastoplastic interactions by means of a finite-discrete element method.
It was shown that the plastic torsional interaction is much stiffer than the elastic interaction. This is due to the larger contact area for plastic interaction under a given normal force. It was also presented that pure slipping occurs at smaller values of angular displacement for plastic interaction. The data presented here confirm the applicability of Deresiewicz's solution for elastoplastic torsional interaction.

The first author would like to express thanks to City, University of London for his doctoral scholarship.

\section{References}

1. J. L. Lubkin, ASME J. Appl. Mech., Vol. 73, pp. 183-187 (1951).

2. H. Deresiewicz, J. Appl. Mech., Vol. 21, pp. 52-56, (1954).

3. C. O'Sullivan, Particulate Discrete Element Modelling: A Geomechanics Perspective. Spon Press (2011).

4. C. Thornton, Granular Dynamics, Contact Mechanics and Particle System simulations, Springer (2015).

5. E. Dintwa, M. van Zeebroeck, E. Tijskens, H. Ramon, Granular Matter 7: 169-179 (2005).

6. S. Nadimi, J. Fonseca, Géotechnique (under review, 2017).

7. A. Munjiza, The Combined Finite-Discrete Element Method, Wiley, London (2004).

8. ABAQUS User's Manual 2016, Dassault Systèmes, version 6.14.

9. S. Nadimi, J. Fonseca, R.N. Taylor, In Deformation characteristics of geomaterials: Proceedings of the $6^{\text {th }}$ International symposium on deformation characteristics of geomaterials, Buenos Aires, Argentina (2015).

10. E. Dintwa, M. van Zeebroeck, E. Tijskens, H. Ramon, Eur. Phys. J. B 39: 77 (2004). 